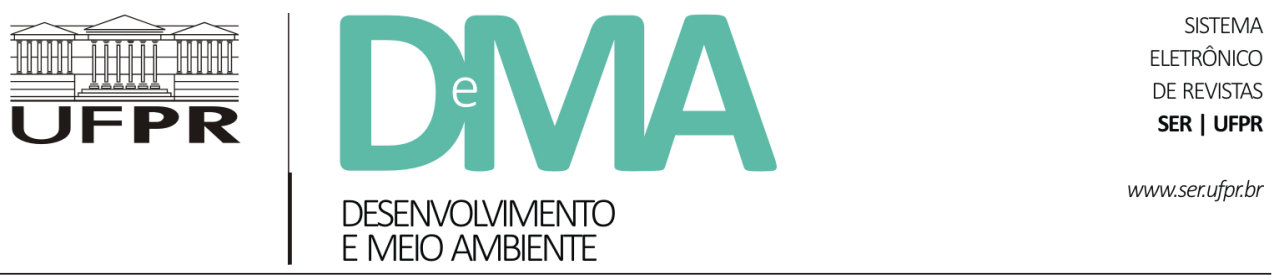

\title{
Avaliação do Programa Nacional de Gerenciamento Costeiro no Brasil: União, Estados e Municípios
}

\section{Evaluation of Coastal Management in Brazil: Union, States and Municipalities}

\author{
Marinez Eymael Garcia SCHERER ${ }^{1 *}$, Milton Lafourcade ASMUS ${ }^{2}$, Tiago Borges Ribeiro GANDRA ${ }^{1,3}$ \\ ${ }^{1}$ Universidade Federal de Santa Catarina (UFSC), Florianópolis, SC, Brasil. \\ ${ }^{2}$ Universidade Federal de Rio Grande (FURG), Rio Grande, RS, Brasil. \\ ${ }^{3}$ Instituto Federal de Educação, Ciência e Tecnologia do Rio Grande do Sul (IFRS), Rio Grande, RS, Brasil \\ *E-mail de contato: marinez.scherer@ufsc.br
}

Artigo recebido em 31 de agosto de 2017, versão final aceita em 24 de janeiro de 2018.

RESUMO: Aavaliação dos processos de gestão é um dos passos previstos na Gestão Costeira Integrada(GCI). Assim, analisouse o Programa Nacional de Gerenciamento Costeiro no Brasil tendo como base publicações que analisaram a estrutura desta gestão no nível federal, no Estado de Santa Catarina e no município de Florianópolis/SC. Estas publicações consideraram os 10 descritores do Decálogo para GCI e os seus resultados foram padronizados para este trabalho. Cada descritor foi avaliado em relação à Existência, à Implementação e à Avaliação Frequente, atribuindo-se um valor de um, para avanço precário, a cinco, para avanço relevante. Os resultados indicam um maior grau de implementação em nível federal, se comparado aos estados e municípios. A alta pontuação dos indicadores quanto à Existência se explica pelo estabelecimento do Plano Nacional de Gerenciamento Costeiro (PNGC) e pela definição dos instrumentos em normas legais. No entanto, a Implementação dos mesmos descritores muitas vezes não ocorre, sendo precária. Destaca-se positivamente a Existência dos descritores Competências, Instituições, Instrumentos e Conhecimento da zona costeira, principalmente para o nível federal. No entanto, destacam-se negativamente os descritores Formação e Capacitação e Recursos, obtendo pontuação baixa nas três esferas de governo. A baixa pontuação para todos os descritores quando avaliados no município é destaque negativo. Em nível local, onde os conflitos emergem e os impactos são evidentes, a atual estrutura e as estratégias de gestão costeira não estão cumprindo seu papel. Aponta-se também que todos os descritores apresentaram pontuação baixa na análise da sua Avaliação e Divulgação. Este resultado aponta para uma falha no ciclo da gestão costeira, pois não existem melhorias em um processo que não é monitorado e avaliado. Assim, este trabalho aponta deficiências na estrutura e nas estratégias de GCI no Brasil, contribuindo para a sua avaliação.

Palavras-chave: gerenciamento costeiro; monitoramento e avaliação; decálogo para a gestão costeira. 
ABSTRACT: One of the steps of an Integrated Coastal Management (ICM) is the evaluation of the whole process. This work assessed the Brazilian Coastal Zone Management Program based on publications exploring the structure of this management at the federal level, in the state of Santa Catarina and local level in the city of Florianópolis /SC. These publications considered the 10 descriptors of the Decalogue for ICM and their results were standardized for the analysis. Each descriptor was evaluated in relation to Existence, Implementation and Frequent Evaluation, assigning a value from one (irrelevant progress) to five (relevant progress). The results indicate a better implementation at Federal level, when compared to States and Municipalities. The establishment of the National Plan for Coastal Management and the definition of the management mechanisms in legal norms explain the high score of the indicators for Existence in the three levels of government. However, the Implementation of the same descriptors often do not occur, as it should. It stands out positively the Existence of the descriptors Competences, Institutions, Instruments and Knowledge of the coastal zone, mainly at Federal level. However, the descriptors Training, Capacity Building and Resources have been negatively assessed, obtaining low scores in the three levels of government. All the descriptors had a low score when evaluated for municipality. At local level, where conflicts emerge and impacts are evident, the current structure and strategies for coastal management are not fulfilling their roles. It is also pointed out that all descriptors evaluated, presented a low score in the analysis of their Frequent Evaluation. This result shows a failure in the coastal management cycle, as it is not possible to improve in a process that is not monitored and evaluated. Thus, this work points out some of the deficiencies in the structure and strategies of ICM in Brazil, contributing to its evaluation.

Keywords: coastal management; monitoring and evaluation; decalogue for coastal management.

\section{Introdução}

A avaliação dos processos de gestão é um dos passos previstos no ciclo da gestão costeira proposto por diversos autores (Cicin-Sain \& Knecht, 1988; Kay \& Alder, 1999; Olsen et al., 1999; Barragán, 2016; Cormier \& Elliot, 2017). Nesta etapa, deve-se verificar se as ações tomadas estão surtindo os efeitos desejados na melhora do ambiente costeiro-marinho e no bem-estar humano das comunidades costeiras. Além das ações operativas, as iniciativas estratégicas da gestão, a própria estrutura que permite uma boa gestão e o envolvimento dos atores, entendidos aqui como governança, devem ser constantemente monitorados. A não avaliação dos processos de gestão costeira dificulta uma gestão adaptativa e a melhoria dos ambientes marinho e costeiro.
O Brasil possui um programa de gestão costeira estruturado desde 1988, com a promulgação da Lei Nacional de Gerenciamento Costeiro (Brasil, 1988). A norma estabelece, com base na Política Nacional de Meio Ambiente (PNMA) e na Política Nacional de Recursos do Mar (PNRM) (Marroni \& Asmus, 2005), o Plano Nacional de Gerenciamento Costeiro (PNGC) (CIRM, 1997). A estrutura de gestão costeira no Brasil conta com competências e instrumentos de planejamento e gestão definidos, permeando as três esferas de governo (Federal, Estadual e Municipal) (Brasil, 1988). No entanto, esta gestão parece não estar implicando em melhoras reais das zonas costeira e marítima brasileira, no tocante à conservação dos sistemas ambientais, dos seus serviços ecossistêmicos e do bem-estar humano (Scherer \& Asmus, 2016). Parte-se do pressuposto de que, apesar do PNGC, a gestão costeira no Brasil 
não vem sendo desenvolvida como estabelecido, em nenhum dos níveis de governo. Assim, este trabalho avaliou a gestão costeira no Brasil nas três esferas de governo, tendo como base publicações que realizaram a análise da governança de gestão costeira.

\section{Método}

A gestão costeira no Brasil foi analisada, comparada e discutida em uma perspectiva do pacto federativo (união, estados e municípios), uma vez que a gestão costeira brasileira é competência destes três níveis de governo. Foram utilizados dados secundários de publicações que realizaram a análise da governança para a gestão costeira no nível federal (Scherer et al., 2009; Scherer et al., 2011), no estado de Santa Catarina (Andrade \& Scherer, 2014) e no município de Florianópolis (Diederichsen et al., 2013). Nestas publicações foram analisados, para cada um dos três níveis de governo, os 10 descritores de gestão (Decálogo para a Gestão Costeira) propostos por Barragán (2004) e utilizados por 13 países da Rede Ibermar (Red Ibermar, 2009). Estes descritos foram também discutidos por Sanabria et al. (2011) e pelas publicações analisadas (Scherer et al., 2009; Scherer et al., 2011; Diederichsen et al., 2013; Andrade \& Scherer, 2014). São eles: 1) Política pública para o litoral; 2) Estrutura Normativa; 3) Competências; 4) Instituições públicas; 5) Instrumentos e Estratégias; 6) Formação e Capacitação; 7) Recursos Econômicos e Financeiros; 8) Informação e Conhecimento; 9) Educação para a Cidadania; 10) Participação cidadã nas tomadas de decisão.

As publicações analisadas utilizaram metodologias e indicadores diferentes, portanto, seus resultados foram harmonizados para permitir uma comparação nos três níveis de governo. Optou-se por padronizar os resultados conforme aqueles apresentados para o Estado de Santa Catarina, atribuindo-se a cada descritor um valor de um (1) a cinco (5), sendo 1 referente a uma situação de avanço precário e 5 como uma situação de avanço relevante. Este critério também foi adotado por Botero \& Sosa (2011) para a análise do Decálogo para a Colômbia.

Além de categorizar os indicadores do decálogo conforme seu avanço, escolheu-se também por integrar a metodologia empregada em Diederichsen et al. (2013), que analisam cada descritor em relação a sua Existência = E; Implementação = I; Avaliação e Divulgação Frequente dos resultados $=$ A.

As publicações referentes ao Brasil (Scherer et al., 2009) utiliza uma análise qualitativa do decálogo e não apresenta indicadores numéricos. Portanto, estes resultados foram avaliados e atualizados para a atribuição dos valores de avanço em relação à Existência, à Implementação e à Avaliação Frequente.

Os resultados referentes a Santa Catarina já se encontravam categorizados numericamente conforme a metodologia aqui apresentada, restando a análise de Existência, Implementação e Avaliação Frequente.

Por fim, a publicação que traz a análise de Florianópolis (Diederichsen et al., 2013) utiliza valores de 1 a 9 para os indicadores de avanço, portanto, estes valores foram reescalonados de forma linear para o intervalo de 1 a 5. Esta publicação apresenta a análise de Existência, Implementação e Avaliação Frequente, sendo utilizada neste trabalho após adaptação dos valores de indicadores de avanço.

Os resultados dos trabalhos avaliados foram colocados em uma tabela comparativa em que cada 
linha representa os descritores do decálogo e cada coluna um nível de governo, sendo calculado para cada caso o percentual da pontuação máxima no conjunto de descritores.

A análise dos resultados da Tabela 1 se deu de maneira discursiva, tomando também como base documentos, tais como as políticas públicas relacionadas à gestão costeira, leis e normas, planos, programas e projetos oficiais, além de literatura científica sobre os descritores analisados.

\section{Resultados e discussão}

A análise dos resultados indica que a união obteve maior avanço na gestão costeira integrada, em seus instrumentos e estratégias, do que o estado e o município, tanto em relação à existência, à implementação e à avaliação e divulgação dos descritores (Tabela 1).
Nota-se que a Existência dos descritores é a mais bem pontuada em todas as esferas, seguida pela Implementação. Os indicadores de avanço em relação à Avaliação e Divulgação das ações têm sempre baixa pontuação, não ultrapassando $30 \%$ da pontuação total nem na esfera Federal.

A alta pontuação de Existência dos descritores analisados se explica pelo estabelecimento do Plano Nacional de Gerenciamento Costeiro (PNGC) e pela definição dos instrumentos estratégicos e operativos, instituídos por força de lei (Lei Federal 7.661/88 - Brasil, 1988; Decreto Federal 5.300/2004 - Brasil, 2004). Entretanto, alguns descritores bem pontuados no quesito Existência tiveram avanços muito pequenos em relação à Implementação.

TABELA 1 - Comparação entre Brasil, Santa Catarina e Florianópolis, analisando os indicadores do decálogo em relação à sua Existência, Implementação e Avaliação.

\begin{tabular}{|c|c|c|c|c|c|c|c|c|c|}
\hline & \multicolumn{2}{|c|}{ Brasil } & \multicolumn{3}{|c|}{ Santa Catarina } & \multicolumn{4}{|c|}{ Florianópolis } \\
\hline & $\mathbf{E}$ & I & $\mathbf{A}$ & $\mathbf{E}$ & I & $\mathbf{A}$ & $\mathbf{E}$ & I & $\mathbf{A}$ \\
\hline Política Pública & 4 & 2 & 1 & 2 & 2 & 1 & 2 & 1 & 1 \\
\hline Competências & 5 & 5 & 2 & 3 & 3 & 2 & 2 & 1 & 1 \\
\hline Instituições & 5 & 4 & 2 & 3 & 2 & 2 & 2 & 2 & 1 \\
\hline Formação e Capacitação & 3 & 3 & 1 & 1 & 1 & 1 & 2 & 1 & 1 \\
\hline Recursos & 2 & 2 & 1 & 2 & 1 & 1 & 1 & 1 & 1 \\
\hline Conhecimento e Informação & 4 & 3 & 2 & 4 & 3 & 3 & 3 & 2 & 1 \\
\hline Educação para a Sustentabilidade & 3 & 2 & 1 & 2 & 2 & 1 & 3 & 3 & 1 \\
\hline
\end{tabular}

Existência $=$ E; Implantação $=$ I; Avaliação e divulgação frequente resultados $=$ A 


\subsection{Política pública para a gestão costeira}

O item de política pública analisado levou em consideração que, em nível federal, a gestão costeira está baseada em duas políticas nacionais (PNRM e PNMA) existentes e implementadas. No entanto, não existe uma política nacional de gestão costeira específica. Alguns estudiosos consideram que o PNGC cumpriria este papel. Mas, ainda que se assuma esta premissa, pode-se notar que o mesmo não está sendo implementado por meio dos instrumentos definidos no próprio PNGC (Scherer et al., 2011; Andrade, 2013).

Já em relação a Santa Catarina e Florianópolis, não se pôde averiguar política pública específica para a gestão costeira, nem tampouco sua implementação, ainda que ambas as esferas possuam uma Lei que institui o Plano Estadual (ou Municipal) de Gerenciamento Costeiro. Sendo assim, tanto o Estado como o município tiveram o valor 2 , pois não possuem política declarada, mas possuem normas que levam à necessidade desta política

\subsection{Normativa para a gestão costeira}

Para o item Normativa específica para a gestão costeira, considerou-se que os três níveis de governo possuem lei de gerenciamento costeiro promulgada, sendo elas: a. Lei Federal 7.661/88 (Brasil, 1988); Decreto Federal 5.300/2004 (Brasil, 2004); b. Lei Estadual 13.553/2005 (Santa Catarina, 2005); Decreto Estadual 5.010/2006 (Santa Catarina, 2006); e c. Lei Municipal 7.975/2009 (Florianópolis, 2009). No entanto, mais uma vez não se verificam as melhorias práticas que deveriam advir destas leis. De uma maneira geral, as leis mencionadas estabelecem a necessidade dos Planos de Gerenciamento Costeiro (nacional, estadual e municipal, respectivamente) e do desenvolvimento de outros instrumentos de gestão. Assim, se os instrumentos não são colocados em prática, como se verá no item 3.4 , a própria lei não está sendo implementada.

\subsection{Competências e instituições}

As competências e as instituições são avaliadas em conjunto, assim como o foram nas publicações de base analisadas. Estes indicadores de avanço alcançaram pontuação máxima de Existência no nível federal e uma pontuação média para o Estado e baixa para o município. As mesmas leis mencionadas acima definem as competências e instituições responsáveis pela gestão costeira em cada escala de governo; sendo assim, elas existem. Importante ressaltar que no estado de Santa Catarina a estrutura administrativa da gestão costeira está alocada em um órgão fora do Sistema Nacional de Meio Ambiente (SISNAMA), condição que contraria a Lei Federal 7.661/88. No que tange à real atuação destas instituições na gestão costeira, o destaque negativo é para o município de Florianópolis, o qual não conta com equipe responsável pela supervisão da implantação do Plano Municipal de Gerenciamento Costeiro (Diederichsen et al., 2013). Já Santa Catarina não possui equipe técnica adequada (Andrade \& Scherer, 2014).

Cabe também destacar a presença de conflitos de competência, especialmente nas áreas da união de uso público e uso comum do povo (praias). Segundo Scherer (2013), este conflito de competências leva a um vazio de gestão e à degradação das nossas praias. Neste sentido, ressalta-se a recente Lei Fe- 
deral 13.240 de 2015, a qual permite a transferência da gestão dos bens da união para os municípios, desde que cumprida uma série de regulamentações explicitadas no Termo de Adesão (Brasil, 2015).

\subsection{Instrumentos e estratégias}

Com relação aos Instrumentos e estratégias adotados pelas três esferas de governo, a análise levou em consideração aqueles instrumentos definidos na Lei Federal 7.661/88 e no Decreto Federal 5.300/04, sendo eles: Plano Nacional de Gerenciamento Costeiro - PNGC; Plano de Ação Federal da Zona Costeira - PAF; Plano Estadual de Gerenciamento Costeiro - PEGC; Plano Municipal de Gerenciamento Costeiro - PMGC; Plano de Gestão da Zona Costeira - PGZC; Sistema de Informações do Gerenciamento Costeiro - SIGERCO; Sistema de Monitoramento Ambiental da Zona Costeira-SMA; Relatório de Qualidade Ambiental da Zona Costeira-RQA-ZC; Zoneamento Ecológico-Econômico Costeiro-ZEEC; Macrodiagnóstico da Zona Costeira - MDZC; e Projeto de Gestão Integrada da Orla Marítima - Projeto Orla.

Como citado acima, os instrumentos definidos para a gestão costeira no Brasil têm implementação deficiente na maioria dos estados costeiros brasileiros e bastante precária nos municípios costeiros. Destaque para o Projeto Orla, o qual aparece como um dos principais instrumentos colocados em prática pelos municípios costeiros, ainda que de forma incompleta, com pouca implementação dos Planos de Gestão Integrados (PGIs) definidos. Dos mais de 400 municípios costeiros brasileiros atuais, 80 já desenvolveram um PGI, sem que existam informações consistentes sobre a implementação e sobre a avaliação do sucesso destas ações (Mafaldo, 2017). Florianópolis se enquadra entre os municípios que iniciaram o processo do Projeto Orla, o qual não foi finalizado e não tem prazo de finalização. Das oito unidades de gestão, nas quais o município foi dividido, somente uma finalizou o PGI. Mesmo assim, o documento não passou pela análise da Comissão Técnica Estadual.

Já em nível estadual, oito dos 17 estados costeiros realizaram o ZEEC, ainda que muitas vezes incompleto ou sem levar em consideração a faixa marítima, e cinco PEGC foram desenvolvidos, mas não implementados (Andrade, 2013). Destaque para Santa Catarina, que realizou o ZEEC de toda a zona costeira, incluindo a porção marítima, e também desenvolveu o Plano Estadual de Gerenciamento Costeiro (SPG, 2010). No entanto, as ações do PE$\mathrm{GC} / \mathrm{SC}$ não foram implementadas, o que requer uma nova avaliação e atualização, passados sete anos.

No que tange aos PMGCs, o desenvolvimento é precário, sendo que se tem notícia de poucos planos desenvolvidos e/ou instituídos por lei municipal, como é o caso de Florianópolis, Joinville, Biguaçu e Itapoá (SC), Campos de Goytacazes (RJ), Anchieta (ES), Salvador (BA). No entanto, nenhum dos PMGCs que existem foram implementados e, muito menos, avaliados.

Em relação às estratégias e aos instrumentos desenvolvidos pela União, destaca-se o Macrodiagnóstico da Zona Costeira e Marinha - MDZCM (MMA, 2009), trazendo informações importantes para a gestão, na escala da união (1:1.000.000). Também se aponta o PAF, o qual teve sua última versão em 2015-16. Deste PAF, apenas 25\% das 16 ações previstas foram realizadas no prazo estabelecido (Coelho, 2017). No momento de escrita deste 
trabalho, está sendo desenvolvido o PAF 2017-2018 (Mafaldo, 2017).

\subsection{Formação e capacitação}

O item Formação e Capacitação tem uma das menores pontuações de toda a análise. Só o item Recursos recebeu uma pontuação geral menor. Este fato não surpreende, uma vez que, na publicação de análise global da região ibero-americana, que utilizou os mesmos descritores aqui apresentados, a lacuna existente na formação e na capacitação de gestores talvez seja um dos problemas mais importantes da gestão costeira na região (Barragán, 2012). No entanto, segundo o mesmo autor, existe uma tendência positiva no aumento de oferta de programas de pós-graduação relacionados ao tema. No Brasil, esta realidade não é diferente, existindo iniciativas federais (ex.: formação dos monitores do Projeto Orla), mas sendo praticamente nulas no estado e no município, não sendo exceção para Santa Catarina e Florianópolis.

Sem gestores capacitados para os desafios da gestão costeira, a expectativa de melhoria do estado dos ambientes costeiro e marinho é de difícil concretização. Segundo Barragán (2012; 2016), gestores capacitados nos aspectos sociais e de resolução de conflito são essenciais para a gestão costeira integrada.

Sobressai-se o Programa de Pós-Graduação em Gerenciamento Costeiro (PPGC) da Universidade Federal de Rio Grande (FURG), primeiro e, até o momento, único programa de pós-graduação inteiramente dedicado ao tema no Brasil. Neste contexto, a concretização do X ENCOGERCO - Encontro Nacional de Gerenciamento Costei- ro, realizado pelo Ministério do Meio Ambiente (MMA) e pelo PPGC/FURG, em junho de 2017, foi um importante fórum de discussão e formação de gestores. Destaque para a reunião dos gestores dos 17 estados costeiros (G17) que se realizou durante o evento (ENCOGERCO, 2017).

\subsection{Recursos}

Com relação aos recursos financeiros existentes para a gestão costeira, cabe destacar o Plano Plurianual Federal (PPA) 2016 - 2019 (Brasil, 2016b), com o seu Programa Temático 2046: Oceanos, Zona Costeira e Antártica. Neste programa, de aproximadamente 500 milhões de reais, estão incluídas ações relacionadas à gestão costeira, como o Planejamento Espacial Marinho e o Projeto Orla. No entanto, este recurso, previsto para quatro anos, é dividido com outras ações vultuosas, como o Plano de Trabalho da exploração de Crostas Cobaltíferas da Elevação de Rio Grande; o levantamento do potencial mineral, biológico e das variáveis oceanográficas da Plataforma Continental Jurídica Brasileira e dos Fundos Marinhos Internacionais; a pesquisa em oceanos e na Antártida (Brasil, 2016b). Assim, estima-se que a quantidade de recursos alocados é insuficiente. Como comparação, há outros programas do PPA 2016-2017, tais como os Programas Temáticos 2078 (Conservação e Uso Sustentável da Biodiversidade), com quase 3 bilhões de reais, 2068 (Saneamento Básico), com 40 bilhões, 2080 (Educação de qualidade para todos), de 200 bilhões, ou o 2015 (Fortalecimento do Sistema Único de Saúde), com 440 bilhões de reais alocados.

Em Santa Catarina e em Florianópolis, não existe registro de recursos financeiros destinados 
diretamente à gestão costeira. No entanto, o Plano Plurianual Federal (PPA) 2016-2017 de Santa Catarina considera o Gerenciamento Costeiro como uma ação da Secretaria de Planejamento (SPG), ainda que não aloque recursos ao programa (SEF, 2017).

Algumas ações, como fiscalização e emissão de licenças ambientais, ordenamento da maricultura, monitoramento da qualidade de água, etc., são custeadas pelos órgãos responsáveis, sem vinculação direta a uma estratégia de gestão integrada das zonas costeira e marinha.

Já os recursos humanos foram avaliados como deficientes em todos os níveis, ainda que no Ministério do Meio Ambiente a equipe esteja definida e atuante, como pôde ser percebido durante o X ENCOGERCO em julho de 2017. No entanto, o quadro de pessoas trabalhando na gestão costeira no Estado de Santa Catarina é bastante reduzido (Andrade \& Scherer, 2014). Já em Florianópolis não existe equipe definida para a gestão costeira integrada (Diederichsen et al., 2013).

\subsection{Conhecimento e informação}

O conhecimento científico da zona costeira e marinha é satisfatório nacionalmente, com várias publicações de interesse, tais como o Atlas Geográfico das Zonas Costeiras e Oceânicas do Brasil (IBGE, 2011); Macrodiagnóstico da Zona Costeira e Marinha - MDZCM (MMA, 2009); Areas Prioritárias para Conservação, Uso Sustentável Repartição de Benefícios da Biodiversidade Brasileira (MMA, 2007); Erosão e progradação no litoral brasileiro (Muehe, 2006), dentre outras. Para o Estado de Santa Catarina, Andrade \& Scherer (2014) apontam lacunas de informações do setor
Sul da zona costeira catarinense, especialmente na porção marinha. Mas, o restante do estado teria informação suficiente para iniciar processos de gestão. Destaque para a recente publicação Metodologia para quantificação de perigos costeiros e projeção de linhas de costa futuras como subsidio para estudos de adaptação das zonas costeiras: litoral norte da Ilha de Santa Catarina e entorno (MMA, 2016a). Florianópolis também possui informações, principalmente do meio terrestre e das áreas das baías. No entanto, existe pouca divulgação fora do meio acadêmico/científico.

O conhecimento das comunidades locais também deve ser levado em consideração em processos de governança (Borrini-Feyerabend et al., 2014), principalmente em uma abordagem ecossistêmica (SCBD, 2004). Neste caso, as três esferas de governo têm poucos estudos relacionados aos conhecimentos tradicionais e menos ainda direcionados às zonas costeira e marinha. Destacam-se iniciativas da Nova Cartografia Social com publicações em Santa Catarina, tais como para a região da Costa da Lagoa em Florianópolis, para o Morro do Boi na Grande Florianópolis (NCS, 2011) e para a região de Imbituba (Mombelli, 2013), entre outras. A experiência do Conselho da APA da Baleia Franca também é digna de nota no que tange à participação e à utilização do conhecimento local para a gestão (Gerhardinger, 2014).

\subsection{Educação para a cidadania}

No quesito Educação para a Cidadania, são poucas as iniciativas de programas e estratégias de educação ambiental relacionadas às zonas costeira e marinha. Em nível nacional, existe o Programa de 
Promoção de Mentalidade Marítima - PROMAR, presente no IX Plano Setorial para os Recursos do Mar - PSRM (Brasil, 2016a). O PROMAR tem como objetivo principal "ter a mentalidade marítima resgatada e fortalecida junto à população brasileira" (Brasil, 2016a). Algumas ações do PROMAR constam no Informativo da Comissão Interministerial para os Recursos do Mar (CIRM, 2017). Destaque para exposições e presença nas redes sociais (Facebook).

Organizações Não Governamentais (ONGs) também contribuem para esse processo de conscientização, como o Programa Bandeira Azul de Gestão e Certificação de Praias (IAR, 2017), a Fundação Brasil Cidadão (2017), Ações do Núcleo de Educação e Monitoramento Ambiental (NEMA, 2017), entre tantas outras.

Ações isoladas e de ONGs são a principal fonte de iniciativas de educação voltadas à conscientização costeiro-marinha no município de Florianópolis, assim como o Projeto Escola do Mar e o Eco-Escolas (Diederichsen et al., 2013). Já o estado de Santa Catarina não tem nenhuma iniciativa oficial voltada a esta conscientização. No entanto, cabe destacar o papel de instituições de ensino, como o Instituto Federal de Santa Catarina, Campus Itajaí, e o Projeto Aprendendo com o Mar (IFSC, 2017), entre outras iniciativas realizadas no estado, mas de abrangência local.

\subsection{Participação}

Por fim, a participação nos processos decisórios e de gestão está prevista em alguns dos instrumentos definidos para a gestão costeira, tais como Planos de Gestão, Zoneamento Ecológi-
co-Econômico e o Projeto Orla. Destaca-se que somente o Projeto Orla define o que seria esta "participação" e dá estratégias de engajamento público, além de prever um Comitê Gestor da Orla participativo (MMA, 2006). Os outros instrumentos da gestão costeira apenas definem a necessidade de participação pública, sem a qualificação da mesma. Sendo assim, a participação muitas vezes se traduz em meras audiências públicas, consultas, sendo estágios preliminares de participação (Arnstein, 1969; Barragán, 2003), os quais não garantem as governanças costeira e marinha. Este padrão é o mesmo em todas as escalas de governo analisadas.

Existem outros espaços de participação nas decisões que afetam as zonas costeiras e marinhas e que não fazem parte dos instrumentos oficiais do Gerenciamento Costeiro brasileiro. Destaque para os Planos Diretores das cidades costeiras, que devem ser desenvolvidos de maneira participativa (Brasil, 2001), e os processos de licenciamento ambiental que requerem audiência pública (CONAMA, 1986). Estes outros instrumentos adicionam processos participativos às tomadas de decisão, mas, a exemplo dos instrumentos do gerenciamento costeiro, a definição desta participação não é explicitada, podendo levar a processos de participação tendenciosa e/ou falha.

Na questão da participação, também deve-se assinalar o Grupo de Integração do Gerenciamento Costeiro (GIGERCO), o qual é coordenado pelo Ministério do Meio Ambiente e pela Comissão Interministerial para os Recursos do Mar (MMA, 2017). Segundo MMA (2017), o GIGERCO tem como objetivos "promover a articulação das ações federais incidentes na zona costeira, a partir do Plano de Ação Federal - PAF-ZC, com vistas a apoiar a implementação do Plano Nacional de 
Gerenciamento Costeiro - PNGC" e conta com a participação de representantes de mais de 30 instituições, incluindo representante das universidades e representante da sociedade civil organizada (ONG). Conforme relatório do último PAF, $63 \%$ dos membros do GIGERCO foram atuantes nas ações do PAF (Coelho, 2017).

Com relação aos descritores acima analisados, a baixa pontuação resultante na coluna de Avaliação e Divulgação é preocupante, uma vez que a gestão costeira integrada segue um ciclo de gestão no qual existem fases de análise do problema, definição e planejamento de ações, monitoramento destas ações, avaliação e implementação de melhorias (Cicin-Sain \& Knecht, 1988; Kay \& Alder, 1999; Olsen et al., 1999; Barragán, 2016). Assim, um processo de gestão no qual ações, instrumentos, planos, programas e políticas implementados não são monitorados e avaliados não permite a gestão adaptativa, ou ainda o fazendo e aprendendo (do inglês, learning by doing). Segundo estes autores, a falta de monitoramento e avaliação frequente, assim como a falta da divulgação dos resultados destas avaliações, enfraquece o processo de gestão e a eficácia do mesmo. Neste sentido, cabe ressaltar o esforço realizado pela sociedade civil em discutir a gestão costeira no Brasil e apontar sugestões de melhoria. Este esforço foi concentrado entre os anos de 2007 e 2009, com a realização dos setes eventos Cidades Costeiras Sustentáveis, cujos principais resultados foram publicados em Scherer et al. (2011). As 167 propostas de ações de melhoria da gestão costeira, aprovadas pelos mais de mil participantes dos eventos, foram enviadas ao Ministério do Meio Ambiente; no entanto, não houve resposta dessa instituição, nem divulgação das propostas.

\subsection{Gestão fora da caixa?}

É importante também destacar que a gerência costeira, no âmbito do Ministério do Meio Ambiente, Gestão Territorial, com apoio do GIGERCO, tem desenvolvido outros projetos relacionados à gestão costeira, mas que não estão descritos no escopo das normas e políticas que estabelecem o gerenciamento costeiro no Brasil. A adição de iniciativas ao escopo original da gestão costeira no Brasil, definida pela Lei Nacional de Gerenciamento Costeiro, é natural e desejável. Após 29 anos da promulgação da lei, outros temas de interesse surgiram, como é o caso da adaptação das áreas costeiras às mudanças do clima, presente na Política Nacional sobre Mudanças do Clima (PNMC).

Dentre as iniciativas da Gerência Costeira em nível federal, cabe ressaltar o Projeto Transferência de Metodologias e Ferramentas de Apoio à Gestão da Costa Brasileira (SMC Brasil), o Programa de Ação Global para a Proteção do Meio Marinho Frente às Atividades Baseadas em Terra (GPA), o Projeto Lixo Marinho e o Grupo de Trabalho em Planejamento Espacial Marinho (MMA, 2017).

Destaca-se também o Projeto Proteção e Gestão Integrada da Biodiversidade Marinha e Costeira - Projeto TerraMar, desenvolvido pelo Ministério do Meio Ambiente em parceria com a GIZ (Gesellschaft für Internationale Zusammenarbeit) do Ministério Federal do Meio Ambiente, Conservação da Natureza, Construção e Segurança Nuclear BMUB, da Alemanha (MMA, 2017).

O Sistema Nacional de Unidades de Conservação (SNUC) (Brasil, 2000) também deve ser identificado como uma forma de gestão territorial, no caso das Unidades de Conservação (UCs) cos- 
teiras e marinhas. Segundo MMA (2010), o Brasil possui, por exemplo, $75,1 \%$ de seus manguezais e $20,7 \%$ dos estuários protegidos por UCs. No entanto, somente $1,5 \%$ das nossas áreas marinhas estão sob algum tipo de proteção (MMA, 2016b). A gestão costeira não pode deixar de reconhecer e trabalhar em conjunto com essas áreas de proteção.

\section{Considerações finais}

Os descritores analisados apontam para a existência de uma estrutura para a gestão costeira nas três esferas de governo brasileiras, mas menos da metade das ações e estratégias necessárias teve avanços em sua implementação e poucas destas são avaliadas e monitoradas.

Destaca-se positivamente a Existência dos descritores Competências, Instituições, Instrumentos, Estratégias e Conhecimento da zona costeira, principalmente para o nível federal. No entanto, destacam-se negativamente os descritores Formação e Capacitação e Recursos, que obtiveram pontuação baixa nas esferas de governo analisadas.

Destaque negativo também para a gestão costeira nos municípios. Assim, nos municípios, onde as ações e impactos se dão diretamente sobre os meios físico e natural, afetando também o socioeconômico, é que existe maior dificuldade de implementação da gestão costeira. Essa situação é preocupante, pois a degradação ambiental e do bem-estar humano ocorrem localmente.

Convém lembrar que a situação atual do gerenciamento costeiro no Brasil não pode ser pensada e analisada de maneira isolada. As origens e causas da falta de implementação eficaz das iniciativas de gestão estão conectas à situação do país, passando pela falta de vontade política, pela precarização das instituições de planejamento e gestão, pelo sucateamento da educação de qualidade, dentre outros problemas de um país em desenvolvimento como o Brasil. Essa análise causal também deve ser objeto de estudo em trabalhos futuros.

Planos, programas e projetos não contribuem, por si só, para uma gestão costeira eficaz. Se não houver implementação real e avaliação do sucesso das ações desenvolvidas, o planejamento e a gestão da zona costeira não irão ser adequados. Além disso, a estrutura de gestão estabelecida, suas estratégias e instrumentos devem ser também avaliados. Após quase 30 anos da Lei Nacional de Gerenciamento Costeiro, essa avaliação geral é necessária, pois temas de interesse e aspirações da sociedade mudam e devem ser levados em consideração. No entanto, conforme Cormier \& Elliot (2017), a gestão deve estar baseada em metas quantificáveis (específicas, mensuráveis, realizáveis, realistas e definidas no tempo), o que não ocorre com o gerenciamento costeiro no Brasil.

Assim, são trazidas aqui algumas das deficiências na estrutura e estratégias de gestão costeira integrada no Brasil, contribuindo para esta necessária avaliação.

\section{Agradecimentos}

Agradecemos a todos os colegas que trabalham em e pesquisam sobre gestão costeira integrada, pelas discussões, trocas de ideias e trabalhos que serviram de consulta para este artigo. 


\section{Referências}

Andrade, J. Gerenciamento costeiro no Brasil: ZEEC e Plano Diretor (estudo de caso - Paulista/PE). Florianópolis, Dissertação (Mestrado Profissional em Planejamento Territorial) - MPPT/FAED/UDESC, 2013.

Andrade, J.; Scherer, M. Decálogo da Gestão Costeira para Santa Catarina: avaliando a estrutura estadual para o desenvolvimento do Programa Estadual de Gerenciamento Costeiro. Desenvolvimento e Meio Ambiente, 29, 2014.

Arnstein, S. R. A Ladder of Citizen Participation. AIP Journal, 35(4), 216-224, 1969. Disponível em: <http://www.planning. org/pas/memo/2007/mar/pdf/JAPA35No4.pdf >.

Barragán, J. M. Medio Ambiente y Desarrollo en Áreas Litorales: introducción a la planificación y gestión integradas. Cádiz-ES: Universidad de Cádiz, Servicio de Publicaciones, 2003. 306 p.

Barragán, J. M. Las Áreas Litorales de España. Del análisis geográfico a la gestión integrada. Barcelona: Editorial Ariel S.A., 2004. 214 p.

Barragán, J. M. (Coord.). Manejo Costero Integrado en Iberoamerica: diagnóstico y propuestas para una nueva política pública. Cádiz-ES: Red IBERMAR (CYTED), 2012. 152 p. ISBN 9788569510186.

Barragán, J. M. Política, gestão e litoral: uma nova visão da Gestão Integrada de Áreas Litorais. Madrid, Espanha: Editorial Tébar Flores, 2016. 685 p.

Borrini-Feyerabend, G.; Dudley, N.; Jaeger, T.; Lassen, B.; Pathak Broome, N.; Phillips, A.; Sandwith, T. Gobernanza de áreas protegidas: de la comprensión a la acción. In: Serie Directrices para buenas prácticas en áreas protegidas, n. 20. Gland, Suiza: UICN XVI, 2014. 123 p.

Botero, C. S.; Sosa, Z. T. Propuestas para la gestión litoral de un país con tres costas: Colombia. In: Barragán Muñoz, J. M. Manejo costero integrado y política pública en Iberoamérica: propuestas para la acción. Cádiz-ES: Red Ibermar (CYTED), 2011. 280 p. ISBN 13: 978-84-694-4844-1.

Brasil. Lei Federal n. 7.661, de 16 de maio de 1988. Institui o Plano Nacional de Gerenciamento Costeiro - PNGC, dispõe sobre regras de uso e ocupação da zona costeira e estabelece critérios de gestão da orla marítima, e dá outras providências. Brasília: DOU de 16/05/1988.

Brasil. Lei Federal n. 9.985, de 18 de julho de 2000. Institui o Sistema Nacional de Unidades de Conservação da Natureza - SNUC. Brasília: DOU de 19.07.2000.

Brasil. Lei Federal n. 10.257, de 10 de julho de 2001. Estatuto das Cidades, 2001. Brasília: DOU de 11.7.2001.

Brasil. Decreto Federal n. 5.300, de 7 de dezembro de 2004. Regulamenta a Lei n. 7.661, de 16 de maio de 1988, que institui o Plano Nacional de Gerenciamento Costeiro-PNGC, dispõe sobre regras de uso e ocupação da zona costeira e estabelece critérios de gestão da orla marítima, e dá outras providências. Brasília: DOU de 8/12/2004.

Brasil. Lei Federal n. 13.240, de 30 de dezembro de 2015. Dispõe sobre a administração, a alienação, a transferência de gestão de imóveis da União e seu uso para a constituição de fundos. Brasília. DOU de 02/06/2016.

Brasil. Lei Federal n. 13.249, de 13 de janeiro de 2016. Aprova o Plano Plurianual 2016-2019. Brasília: DOU de 14/01/2016. 2016a.

Brasil. Decreto Federal n. 8.907, de 22 de novembro de 2016. Aprova o IX Plano Setorial para os Recursos do Mar. Brasília: DOU de 23/11/2016. 2016b.

Cicin-Sain, B.; Knecht R. W. Integrated Coastal and Ocean Management: Concepts and Practice. Washington, DC: Island Press, 1988. 517 p.

CIRM - Comissão Interministerial para os Recursos do Mar. Resolução 05 de 1997. Aprova o Plano Nacional de Gerenciamento Costeiro II (PNGC II). Brasília: DOU de 1997.

CIRM - Comissão Interministerial para os Recursos do Mar. InforCirm. Brasília, 2017. Disponível em: <https://www.mar. mil.br/secirm/publicacoes/infocirm/2017/ago/index.html $>$.

Coelho, A. Balanço do PAF-ZC 2015-2016. Apresentação. Departamento de Gestão Ambiental Territorial (DGAT). Secretaria de Recursos Hídricos e Qualidade Ambiental (SRHQ). Ministério do Meio Ambiente, 2017.

CONAMA - Conselho Nacional do Meio Ambiente. Resolução 01/1986. Estabelece as definições, as responsabilidades, os 
critérios básicos e as diretrizes gerais para uso e implementação da Avaliação de Impacto Ambiental como um dos instrumentos da Política Nacional do Meio Ambiente. DOU de 17 /2/1986.

Cormier, R.; Elliot, M. SMART Marine Goals, Targets and Management - is SDG 14 opertaional or aspirational, is 'Life Below Water' sinking or swimming? Marine Pollution Bulletin, 2017. doi: 10.1016/j.marpolbul.2017.07.060

Diederichsen, S. D.; Gemael, M. K.; Hernande, A. de O.; De Oliveira, A. de O.; Paquette, M.; Schmidt, A. D.; Silva, P. G. da; Silva, M. S. da; Scherer, M. Gestão costeira no município de Florianópolis, SC, Brasil: um diagnóstico. Revista da Gestão Costeira Integrada, 13(4), 499-512, 2013.

ENCOGERCO - Encontro Nacional de Gerenciamento Costeiro. Disponível em: <http://www.encogerco.com.br>. Acesso em: jun. 2017.

Florianópolis. Lei n. 7975/2009, de 02 de outubro de 2009. Institui o Plano Municipal de Gerenciamento Costeiro. DOM de 27/10/2009.

Fundação Brasil Cidadão. Disponível em: <http://www.brasilcidadao.org.br>. Acesso em: jul. 2017.

Gerhardinger, L. C. Inovação, governabilidade e protagonismo de pessoas-chave na Área de Proteção Ambiental da Baleia Franca (Santa Catarina, Brasil). Campinas, Tese (Doutorado) - Núcleo de Estudos e Pesquisas Ambientais e Instituto de Filosofia e Ciências Humanas da Universidade Estadual de Campinas (IFCH/NEPAM/UNICAMP), 2014.

IAR - Instituto Ambientes em Rede. Programa Bandeira Azul. Disponível em: <http://www.bandeiraazul.org.br>. Acesso em: jul. 2017.

IBGE - Instituto Brasileiro de Geografia e Estatística. Atlas Geográfico das Zonas Costeiras e Oceânicas do Brasil. Rio de Janeiro: IBGE, Diretoria de Geociências. 2011. 176p. ISBN 978-85-240-4219-5. Disponível em: <http://biblioteca. ibge.gov.br/visualizacao/livros/liv55263.pdf $>$. Acesso em: ago. 2017.

IFSC - Instituto Federal de Santa Catarina. Projeto Aprendendo com o Mar. Disponível em: <http://www.ifsc.edu.br/ campus-itajai/5806-projeto-aprendendo-com-o-mar-promove-primeira-aula-de-consciencia-ambiental-no-barco-escola> . Acesso em: jul. 2017.
Kay, R.; Alder, J. Coastal Planning and Management. New York: E \& FN SPON - Routledge, 1999. 375 p.

Mafaldo, S. Comunicação pessoal. Gerência Costeira, Ministério do Meio Ambiente, Brasília, DF. 2017.

Marroni, E. V.; Asmus, M. L. Gereciamento Costeiro: uma proposta para o fortalecimento comunitário na gestão ambiental. Pelotas: Editora USEB, 2005. 147 p.

MMA - Ministério do Meio Ambiente. Projeto Orla: fundamentos para a gestão integrada. 74 p. Ministério do Meio Ambiente (MMA) / Ministério do Planejamento, Orçamento e Gestão, Brasília, DF, Brasil, 2006. 74 p. ISBN: 8577380297. Disponível em: $<$ http://www.mma.gov.br/estruturas/orla/_arquivos/11_04122008111238.pdf>.

MMA - Ministério do Meio Ambiente. Áreas prioritárias para conservação, uso sustentável e repartição de beneficios da biodiversidade brasileira: Atualização - Portaria MMA n. 9, de 23 de janeiro de 2007. / Ministério do Meio Ambiente, Secretaria de Biodiversidade e Florestas. Brasília, MMA, 2007. 300 p. ISBN 978-85-7738-076-3.

MMA - Ministério do Meio Ambiente. Macrodiagnóstico da Zona Costeira e Marinha do Brasil. Ministério do Meio Ambiente, Secretaria de Mudanças Climática e Qualidade Ambiental, Departamento de Qualidade Ambiental, Gerência de Qualidade Costeira e Marinha. Brasília, 2009. 242 p.

MMA - Ministério do Meio Ambiente. Panorama da Conservação dos Ecossistemas Costeiros e Marinhos no Brasil. Secretaria de Biodiversidade e Florestas, Gerência de Biodiversidade Aquática e Recursos Pesqueiros. Brasília, 2010. 148 p. ISBN 978-85-7738-142-5.

MMA - Ministério do Meio Ambiente. Metodologia para quantificação de perigos costeiros e projeção de linhas de costa futuras como subsídio para estudos de adaptação das zonas costeiras: litoral norte da Ilha de Santa Catarina e entorno. Ministério do Meio Ambiente. Secretaria de Extrativismo e Desenvolvimento Rural Sustentável; Laboratório de Oceanografia Costeira; Universidade Federal de Santa Catarina; Elaboração: Klein, A. H. da F. et al. Brasília, 2016a. ISBN: 978-85-7738-266-8. Disponível em: <http://www.mma.gov.br/ images/arquivo/80247/Publicacoes/LIVRO_LITORAL\%20 NORTE\%20SC_WEB.pdf>. Acesso em: jul. 2017.

MMA-Ministério do Meio Ambiente. $5^{\circ}$ Relatório Nacional 
para a Convenção sobre Diversidade Biológica. Secretaria de Biodiversidade e Florestas. Coordenador: Scaramuzza, C. A. de M. Brasília, 2016b. 240 p.

MMA - Ministério do Meio Ambiente. Gestão Territorial. Disponível em: <http://www.mma.gov.br/gestao-territorial >. Acesso em jul. 2017.

Mombelli, R. Comunidade tradicional dos Areais da Ribanceira, Imbituba (SC): desenvolvimento, territorialidade e construção de direitos. Estudos de Sociologia, 18(35), 325-334, 2013.

Muehe, D. (Org.). Erosão e progradação no litoral brasileiro. Brasília: MMA, 2006. 476 p. ISBN 85-7738-028-9.

NCS - Nova Cartografia Social dos Povos e Comunidades Tradicionais do Brasil. Quilombolas do Morro do Boi, Santa Catarina. Organizador: Almeida, A. W. B. de. Manaus: Projeto Nova Cartografia Social da Amazônia/UEA Edições, 2011.

NEMA - Núcleo de Educação e Monitoramento Ambiental. Disponível em: $<$ http://www.nema-rs.org.br>. Acesso em: jul. 2017.

Olsen, S. B.; Lowry, K.; Tobey, J. A Manual for Assessing Progress in Coastal Management. The University of Rhode Island. Coastal Resources Center, Graduate School of Oceanography, Narragansett, RI 02882 USA, 1999. 56 p. Disponível em: $<$ http://www.commissionoceanindien.org/fileadmin/resources/ RECOMAP\%20Manuals/Manual\%20for\%20Assessing\%20 Progress\%20in\%20Coastal\%20Management\%202009.pdf .

Red Ibermar - Rede Iberoamericana de Manejo Costero Integrado. Manejo costero integrado y política pública en Iberoamérica: un diagnóstico. Necesidad de cambio. Coordenador: Barragán Munõz, J. M. Editores: Granados, P. A.; Chica Ruiz, J. A.; Onetti, J. G.; Sanabria, J. G. Espanha: CYTED-SEGIB, 2009. 380 p.

Sanabria, J. G.; Onetti, J. G.; Barragán, J. M. M. Las comunidades autónomas y la gestión integrada de las áreas litorales de España. Materiales para un debate sobre gobernanza. Cádiz-ES: Grupo de Investigación en Gestión Integrada de Áreas Litorales de la Universidad de Cádiz, 2011.337 p. ISBN: 978-84-695-1402-3.

Santa Catarina. Lei $n^{\circ} 13.553$, de 16 de novembro de 2005. Institui o Plano Estadual de Gerenciamento Costeiro. Palácio Barriga Verde. Florianópolis, SC, 2005.
Santa Catarina. Decreto $n^{\circ}$ 5.010, de 22 de dezembro de 2006. Regulamenta a Lei ${ }^{\circ} 13.553$, de 16 de novembro de 2005. Florianópolis, 22 de dezembro. DOSC de 22.12.2006.

SCBD - Secretariat of the Convention on Biological Diversity. The Ecosystem Approach (CBD Guidelines). Montreal: Secretariat of the Convention on Biological Diversity, 2004. 50p.

Scherer, M. Gestão de Praias no Brasil: subsídios para uma reflexão. Revista de Gerenciamento Costeiro Integrado, 2013. doi: $10.5894 /$ rgci358

Scherer, M.; Sanches, M.; Negreiros, D. H. Gestão de Zonas Costeiras e as Políticas Públicas no Brasil: um diagnóstico. In: Manejo Costero Integrado y Política Pública en Iberoamérica: un diagnóstico. Necesidad de cambio. Ed. Red Iberoamericana de Manejo Costero - Red Ibermar (CYTED), 2009. 377 p.

Scherer, M.; Asmus, M.; Filet, M.; Sanches, M.; Poleti, A. E. El manejo costero en Brasil: análisis de la situación y propuestas para una posible mejora. In: Farinós Dasí, J. (Ed. y Coord.). La Gestión Integrada de Zonas Costeras ¿Algo Más que una Ordenación del Litoral Revisada? La GIZC como evolución de las prácticas de planificación y gobernanza territoriales. Valencia: PUV/IIDL, 161-173, 2011.373p. (Colección "Desarrollo Territorial", 9). ISBN: 978-84-370-8009-3.

Scherer, M.; Asmus, M. L. Ecosystem-Based Knowledge and Management as a tool for Integrated Coastal and Ocean Management: A Brazilian Initiative. In: Vila-Concejo, A.; Bruce, E.; Kennedy, D.M.; McCarroll, R. J. (Eds.). Proceedings of the 14th International Coastal Symposium (Sydney, Australia). Journal of Coastal Research, Special Issue, 1(75), 2016.

SEF - Secretaria de Estado da Fazenda de Santa Catarina. Plano Plurianual 2016-2019. 2017. Disponível em: <http:// www.sef.sc.gov.br/arquivos_portal/relatorios/77/PPA_Perfil_dos_Orgaos.pdf>. Acesso em: ago. 2017.

SPG - Secretaria de Planejamento do Estado de Santa Catarina. Programa Estadual de Gerenciamento Costeiro, 2010. Disponível em: <http://www.spg.sc.gov.br/acoes/20-gerco> Acesso em ago. 2017. 